\title{
Hepaticojejunostomy leak after pancreaticoduodenectomy
}

\author{
Hui Qiu*, Ji Zhang, Hong-Gang Qian, Jia-Hua Leng, Jian-Hui Wu, Bo-Nan Liu, Qiao Liu, \\ Ang Lv , Chun-Yi Hao
}

\begin{abstract}
Department of HPB Surgery, Peking University Cancer Hospital and Institute, Key Laboratory of Carcinogenesis and
\end{abstract} Translational Research (Ministry of Education), Beijing, China

Received: 27 December 2015

Revised: 12 February 2016

Accepted: 29 February 2016

\author{
*Correspondence: \\ Dr. Qiu hui, \\ E-mail: qiuhuibz@163.com
}

Copyright: ( ) the author(s), publisher and licensee Medip Academy. This is an open-access article distributed under the terms of the Creative Commons Attribution Non-Commercial License, which permits unrestricted non-commercial use, distribution, and reproduction in any medium, provided the original work is properly cited.

\begin{abstract}
Background: Hepaticojejunostomy (HJ) leak is the second common anastomotic failure after pancreaticoduodenectomy (PD), but only a few studies have focused on this complication. We evaluated the incidence of HJ leak after PD and described its presentation, treatment, and outcome.

Methods: Records of 292 consecutive patients who underwent PD between 2007 and 2014 were retrospectively analysed. Clinicopathologic data were compared with patients without HJ leaks, and presentation, radiologic findings, treatment, and outcome of HJ leaks were analysed.

Results: HJ leak was identified in $14(4.8 \%)$ patients. Low serum albumin on postoperative day 1 (POD1) was associated with an increased risk, while dilation of common hepatic duct and preoperative biliary decompression were two protected factors. Median postoperative day of diagnosis was 5 (range, 1-15). Typical clinical signs included bilious drainage in the surgically placed drains, with fever, abdominal pain and leukocytosis. Patients with HJ leaks had more pancreatic fistulas and other complications included Intra-abdominal abscess, wound infection and delayed gastric emptying. $4(29 \%)$ patients were treated operatively, 5 required percutaneous drainage and 5 underwent conservative management. One patient died in hospital, resulting in a mortality of $7 \%$.

Conclusion: Hepaticojejunostomy leaks are rare after PD. The complication severity ranges from trivial to life threatening. It may be associated with nutritional status, width of common bile duct and surgical procedures. Surgical interventions are required for more than half of HJ leaks. A good outcome can be expected.
\end{abstract}

Keywords: Hepaticojejunostomy leak, Pancreaticoduodenectomy, Risk factors

\section{INTRODUCTION}

Pancreaticoduodenectomy (PD) is still the first-line procedure for most tumors localized at the periampulla. The reconstruction usually contains 3 anastomoses: pancreaticojejunostomy (PJ)/pancreaticogastrostomy (PG), hepaticojejunostomy (HJ) and gastrojejunostomy. Although the mortality rate for PD has dramatically dropped for almost $20 \%$ in recent years, but the morbidity rate remains over $50 \%$ even at the high-volume centers. HJ leak is the second common anastomoses deficiency, occurring in $2.2-12 \%$ of patients. $^{1-4}$ Unlike the postoperative pancreatic fistula (PJ or PG leak) which have been paid more attention in the literatures, the reports about HJ leak are rare, though the clinical sequelae may also be life threatening.

Searching articles on PubMed, only a few studies focusing on HJ leaks after PD have been published. And the severity ranges from trivial to life threatening. Therefore, the aim of the present study was to define its incidence, presentation, risk factors and management options. ${ }^{1-3,5,6}$ 


\section{METHODS}

Data were obtained from a prospectively maintained departmental pancreatic surgery database. All patients who underwent PD from 2007 to 2014 in Department of HPB II (Beijing Cancer Hospital, Beijing, China) were reviewed, and a total of 292 consecutive PDs were performed (or supervised) by a single surgeon in the study period.

Patients were dichotomized according to whether or not they experienced a postoperative $\mathrm{HJ}$ leak, and the information's were recorded to analyze HJ leak risk factors, including patient demographics, placement of a biliary drainage catheter, laboratory results, intraoperative data and other postoperative complications.

The relevant information, such as interval between PD and HJ leak, grade of HJ leak, details of management and outcome were also presented.

\section{Definition}

The definition of some complications has been described elsewhere, such as postoperative pancreatic fistula (POPF), massive bleeding, and Intra-abdominal abscess. Surgical mortality was defined as a postoperative death within thirty days.

In all cases, HJ leak was confirmed by imaging with a contrast study through an abdominal drainage tube, which was either placed in the initial PD operation or inserted percutaneously by radiology intervention postoperatively. Clavien-Dindo classification of complications was used to quantify the severity of HJ leak.

According to the happening period either on the first 72 hours or after it, we defined the HJ leak as "early" or "late". And if the volume of bile-rich drainage was more than $250 \mathrm{ml} /$ day, we defined it as "high-output" HJ leak.

\section{Surgical techniques}

The common hepatic duct (CHD) was divided sharply at the level above the cystic duct just after the hepatitis lymphadenectomy. And the blood supply of the stump was protected carefully by remaining some surrounding tissues. If a visible bleeding was found from the marginal arteries, it usually could be sutured.

After the pancreaticogastrostomy (using an invagination technique) and gastrojejunostomy (usually two-layered hand sewn technique) were completed, A Roux limb of the jejunum loop was brought to the level of the CHD retro colic to the right of the middle colic vessels through the avascular area of the mesentery. The size of the opening in the antimesenteric side of the jejunum was accorded with the outside diameter of the CHD, and usually smaller than it.
The anastomoses of the CHD and the Roux limb were performed in an end to side fashion with 5-0 PDS. Two stay sutures were placed at the anterior wall of the anastomoses respectively to expose the posterior clearly, and another stay suture was placed at one corner, then the full-thickness of the hepatic duct and serosa of the bowel wall were sutured continuously from the other corner. The posterior line of the suture was placed first and the knots were tied outside the lumen after the anterior line was finished. No intraluminal drainage was placed regardless the diameter of the CHD.

Two silicone drains were placed near the $\mathrm{HJ}$ and $\mathrm{PG}$ anastomoses. One was introduced from the right side of the incision, with the tip underneath the HJ and the side holes at the sub hepatic space. The other was introduced from the left, with the tip at the lesser sac and the side holes near the PG and gastrojejunostomy.

If no anastomoses associated complications (such as PF or HJ leak) occurred, the drainage should be removed on postoperative day 3 or 5 with the discretion of the operation surgeon.

\section{Statistical analysis}

Comparisons between patients, with and without an $\mathrm{HJ}$ leak, were performed, using a univariate analysis to determine factors predictive of HJ leak. Averages were provided as median values. Continuous values were analyzed using a Wilcoxon rank-sum test, and categorical variables were analyzed using a Fisher's exact test. Because of the low number of events, multivariate analysis for the identification of possible risk factors for HJ leak was not feasible. Statistical significance was accepted for $\mathrm{p}<0.05$ in a two-tailed analysis.

\section{RESULTS}

A total of 292 patients who received PD were selected for this study. Fourteen $(4.8 \%)$ patients developed postoperative HJ leaks.

The Demographic and clinicopathologic data of HJ and Non-HJ groups are summarized in Table 1. The two groups showed no significant differences in age or sex. Previous medical history and body mass index were not different between the 2 groups. Also, there was no difference between preoperative serum albumin. However, a decreased serum albumin on the first postoperative day (POD1) was found to be associated with HJ leak. The median albumin on POD1 in the HJ leak group was $27.7 \mathrm{~g} / \mathrm{l}$, as compared to $33.9 \mathrm{~g} / \mathrm{l}$ in the no leak group $(\mathrm{p}=0.005)$. The rate of dilation of common hepatic duct in patients with HJ leakage was significantly lower than no leakage group (43\% vs $71 \%$; $\mathrm{P}=0.029)$. However, the rate of preoperative biliary decompression was no difference between two groups. 
Table 1: Characteristics of 292 patients who underwent pancreaticoduodenectomy: HJ leak vs. non-HJ leak.

\begin{tabular}{|c|c|c|c|}
\hline Characteristic & HJ leak $(n=14)$ & Non-HJ leak $(\mathrm{n}=278)$ & p value \\
\hline \multicolumn{4}{|l|}{ Patient variables } \\
\hline Age, years, median (range) & $56(42-69)$ & $61(18-86)$ & 0.392 \\
\hline Males, $\mathrm{n}(\%)$ & $10(71)$ & $162(58)$ & 0.06 \\
\hline \multicolumn{4}{|l|}{ Comorbidity } \\
\hline History of diabetes mellitus, $\mathrm{n}(\%)$ & 3 & 45 & 0.412 \\
\hline History of hypertension, $\mathrm{n}(\%)$ & 2 & 38 & 0.596 \\
\hline Body-mass index & $24.5 \pm 4.5$ & $24.2 \pm 3.8$ & 0.155 \\
\hline Dilation of common hepatic duct, n (\%) & $6(43)$ & $198(71)$ & 0.029 \\
\hline Preoperative biliary decompression, n (\%) & $4(29)$ & $87(31)$ & 0.546 \\
\hline \multicolumn{4}{|l|}{ Laboratory results } \\
\hline Albumin Preop, g/L, median (range) & $44.3(33.3-52.4)$ & $43.8(32.9-53.4)$ & 0.481 \\
\hline Albumin on POD1, g/L, median (range) & $27.7(23.3-34.9)$ & $33.9(19.0-41.3)$ & 0.005 \\
\hline \multicolumn{4}{|l|}{ Intraoperative data } \\
\hline Multivisceral resection, $\mathrm{n}(\%)$ & $4(29)$ & $20(7)$ & 0.02 \\
\hline SMV/PV reconstruction, $\mathrm{n}(\%)$ & $2(14)$ & $35(13)$ & 0.55 \\
\hline IBL, mL, median (range) & $400(200-3500)$ & $400(100-3000)$ & 0.208 \\
\hline Operating time, min, median (range) & $450(300-700)$ & $500(270-660)$ & 0.175 \\
\hline Solution volumes transfused, mL, median (range) & $6500(4500-11000)$ & $6000(4000-10000)$ & 0.184 \\
\hline \multicolumn{4}{|l|}{ Pathologic characteristics } \\
\hline Malignant n (\%) & $14(100)$ & $263(95)$ & 0.47 \\
\hline Benign $\mathrm{n}(\%)$ & $0(0)$ & $15(5)$ & \\
\hline
\end{tabular}

Preop preoperation, POD postoperative day, SMV/PV superior mesenteric vein/ portal vein, IBL intraoperative blood loss.

Table 2: Outcomes of 292 patients who underwent pancreaticoduodenectomy: HJ leak vs. non-HJ leak.

\begin{tabular}{|c|c|c|c|}
\hline Characteristic & HJ leak(n=14) & Non-HJ leak(n=278) & p value \\
\hline Any complication, n (\%) & $14(100)$ & $125(45)$ & 0.000 \\
\hline \multicolumn{4}{|l|}{ Operative complications } \\
\hline Pancreatic fistula ${ }^{\#}, \mathrm{n}(\%)$ & $5(36)$ & $31(11)$ & 0.019 \\
\hline Gastrojejunostomy leakage, n (\%) & $1(7)$ & $6(2)$ & 0.294 \\
\hline Intra-abdominal abscess, n (\%) & $5(36)$ & $30(11)$ & 0.017 \\
\hline Wound infection, $\mathrm{n}(\%)$ & $3(21)$ & $11(4)$ & 0.023 \\
\hline Delayed gastric emptying*, n (\%) & $5(36)$ & $29(10)$ & 0.015 \\
\hline Massive bleeding, $\mathrm{n}(\%)$ & $2(14)$ & $26(9)$ & 0.396 \\
\hline Nonoperative complications & $5(36)$ & $52(19)$ & 0.114 \\
\hline Reoperation, n (\%) & $4(29)$ & $16(6)$ & 0.010 \\
\hline Perioperative mortality, n (\%) & $1(7)$ & $8(3)$ & 0.361 \\
\hline
\end{tabular}

\#Grade B or C according to the consensus definition by the International Study Group of Pancreatic Fistula; *Grade B or C according to the consensus definition by the International Study Group of Pancreatic Surgery.

Most intraoperative factors were similar, such as intraoperative blood lose, operating time, and solution volumes transfused. However, additional resection procedures were significantly more often performed in patients with HJ leakage (4[29\%] vs 20 [7\%]; P = .02); Four patients in HJ leak group underwent multivisceral resection (2 hemihepatectomy, and 2 right hemicolectomy). There was no difference in SMV/PV reconstruction between groups.
The rates of resection for malignancy were also similar. The pathologic diagnoses in HJ leak group included six ampullary adenocarcinomas, six pancreatic ductal adenocarcinomas and two neuroendocrine carcinomas.

Table 2 displays the postoperative complications, number of reoperations and perioperative mortality of patients with and without HJ leak. Patients in the HJ leak group were more likely to have postoperative pancreatic fistula (36 vs. $11 \%$ in non-HJ leak, $\mathrm{p}=0.019$ ), intra-abdominal abscess ( 36 vs. $11 \%, \mathrm{p}=0.017$ ), wound infection ( 21 vs. 
$4 \%, \mathrm{p}=0.023$ ) and delayed gastric emptying (36 vs. $10 \%$, $\mathrm{p}=0.015)$. There were no differences in massive bleeding and non-operative complications.

Reoperations were performed in 4 patients in HJ leak group, significantly more than in patients without HJ leak (29\% vs 6\%; $\mathrm{P}=0.010)$. Perioperative mortality was similar between two groups (7\% vs $3 \% ; \mathrm{P}=0.361)$, and only 1 patient died in-hospital in HJ leak group.

Table 3 displays the clinical presentation of patients with HJ leak. Median postoperative day of diagnosis of HJ leak was 5 (range 1-15).The most common clinical sign was bilious drainage (100\%) in the drains either placed in the initial operation or inserted postoperatively, followed by fever $(71 \%)$ and abdominal pain $(50 \%)$. Thirteen patients had leukocytosis (defined as WBC>10,000 cells $/ \mu \mathrm{l})$, with a median white blood cell count was $14.8^{*}$ 109/1. Radiographic evidence of an HJ leak could be obtained in all patients by sinogram or percutaneous trans hepatic cholangiogram.

Table 3: Presentation of $\mathbf{1 4}$ patients with $\mathrm{HJ}$ leak after pancreaticoduodenectomy.

\begin{tabular}{|ll|}
\hline Characteristic patients with HJ leak $(\mathrm{n}=14)$ \\
\hline Median POD of diagnosis (range) & $5(1-15)$ \\
\hline Clinical presentation & \\
\hline Bilious drainage, $\mathrm{n}(\%)$ & $14(100)$ \\
\hline Abdominal pain, $\mathrm{n}(\%)$ & $7(50)$ \\
\hline Fever, $\mathrm{n}(\%)$ & $10(71)$ \\
\hline Leukocytosis, $>10,000$ cells $/ \mu \mathrm{L}, \mathrm{n}(\%)$ & $13(93)$ \\
\hline Radiographic evidence, $\mathrm{n}(\%)$ & $14(100)$ \\
\hline
\end{tabular}

With regards to treatment, one patient simply required prolonged abdominal drainage without the need for pharmacological treatment. Four patients required intravenous administration of antibiotics without other interventions. All of the remaining 9 patients underwent either a percutaneous image-guided technique to optimize drainage or a percutaneous trans hepatic biliary drainage catheter placement. In four of these patients, reoperations were required, because of serious intra-abdominal infection. Two patients developed massive bleeding and received trans catheter arterial embolization. After the haemostasis, both of them received reoperation to eliminate the cause of bleeding.

According to Clavien-Dindo classification of complications, five patients were graded I or (conservative management), eight patients were grade III or IV (requiring an intervention or Intensive Care Unit management), and one patient was grade V (death).

\section{DISCUSSION}

HJ leak is one of the uncommon complications after PD. Unlike pancreatic fistula, in many studies about PD, the HJ leak has been mentioned just by name. Searching on
PubMed, we could find only a few articles focusing on HJ leak after PD. The first one was published in 2003, and no perioperative risk factors were established except for the anastomotic leak test. Another one was published in 2013 and low preoperative albumin was concluded as the only negative risk factor. ${ }^{1,2}$

In the present study, we have experienced a total of $14 \mathrm{HJ}$ leaks out of 292 consecutive PD operations, and the leak rate is $4.8 \%$. Several perioperative factors are demonstrated to be associated with HJ leak in univariate statistical analysis, including dilation of common hepatic duct, serum albumin level on POD1 and multivisceral resection. Multivariable analysis for the identification of risk factors was not possible owing to the low number of events, so it cannot be concluded that these are true risk factors for HJ leak.

Preoperative hypoalbuminaemia was proved to be an independent predictor of poor perioperative outcomes for patients in several malignancies. In this series most of our patients in each group seem to have normal nutrition status before the operation. But some important markers were not tested, such as serum prealbumin and etc. So we designed a new screening system to evaluate the nutritional status and offered nutritional support before the operation. ${ }^{2,7,8}$

Every patient in HJ leak group had experienced hypoalbuminaemia on the first postoperative day (23.3$34.9 \mathrm{~g} / \mathrm{L}$ ), and the median albumin level is significant lower than non-HJ leak group patients' (27.7vs33.9g/L). And postoperative low albumin level might lead intestinal wall edema which could make the HJ anastomose more fragile to rupture, and the mucosa edema could cause increased intraluminal pressure which may lead to HJ leak. The reported reasons for postoperative hypoalbuminaemia included surgery duration, preoperative serum albumin, and solution volumes transfused intra-operatively. But these intraoperative associated factors are not different between the two groups. ${ }^{9}$

In this study a total of 204 patients had dilated common hepatic ducts preoperatively, defined as the diameter more than $1 \mathrm{~cm}$, measured at the level of $1 \mathrm{~cm}$ below the bifurcation on CT or MRI scans. And the dilation can reduce the risk of HJ-leak, because the large diameter biliary duct can make the anastomoses technically easier. In addition round sutures are routine used for all the hepaticojejunostomy in our center, and this continuous type prefers large size of common bile duct, because it will be difficult to keep a suitable distance of suture when the diameter of the duct is very small.

Sometimes the patients with obstructive jaundice should wait for the PD operation more than one month, and such a long period of hyperbilirubinemia would deteriorate their nutritional, metabolic and immune functions. So the preoperative biliary decompression was performed for 
these patients and it seemed that this procedure could not improve HJ leaks. In spite of this, we do not recommend it as a routine procedure before PD because of the high risk of other infection complications. A recent metaanalysis also concluded that middle-distal obstruction in patients who were candidates for PD did not usually require routine biliary drainage. ${ }^{10}$

To achieve margin-negative resection, more patients in $\mathrm{HJ}$ leak group required multivisceral resections(MVR) at the time of PD than non-HJ leak group (29\% vs $7 \%$ ), which would necessarily increase the complexity of the PD operation and the postoperative complications. A recent meta-analysis including a total of $9927 \mathrm{PD}$ demonstrated that MVR was associated with 3-fold mortality and substantial morbidity, and colectomy was an independent predictor of increased overall morbidity with a leading complication of organ-space surgical infection. ${ }^{11}$ Another factor about the MVR associated HJ leak was hepatectomy. Liver resection with biliary reconstruction was widely adopted for the resection of hilar cholangiocarcinoma, and sometimes for a small part of primary tumor located at specific site of the liver. The reported bile leak rate for this procedure was higher than the liver resection alone, and major hepatectomy and lymphadenectomy were considered as risk factors. ${ }^{12,13} \mathrm{In}$ our center the hepatoduodenal ligament lymph node dissection was performed routinely for malignancy and the extrahepatic biliary duct was skeletonized, which would destroy the blood supply of common hepatic duct partially. Meanwhile liver resection, especially for right hepatectomy, would make a further diminution of blood supply and prevent the healing of $\mathrm{HJ}$ anastomoses.

$\mathrm{PD}$ with $\mathrm{PV} / \mathrm{SMV}$ reconstruction was proved to be a safe and reliable treatment, but the vascular stenosis or thrombus could not be completely avoided. ${ }^{14}$ This complication would result in congestive edema of the intestinal wall and sometimes might be lethal, especially for acute thrombus occurred early after surgery. A total of 37 patients underwent this procedure in this series with no significant difference in the proportion between $\mathrm{HJ}$ leak and non-HJ leak groups. However, one patient in HJ leak group developed acute thrombus on POD1, followed by rupture of the anterior wall of $\mathrm{HJ}$ anastomoses and bile peritonitis. After reoperation on POD5, the serious infection was temporary under control, but vascular thrombus could not be completely solved. Then the pancreatic fistula and gastrointestinal anastomotic leakage occurred, and patient died for septic shock and multiple organ failure.

Bilious drainage was a typical presentation of HJ leak, and usually observed in the early postoperative period. Other nonspecific clinical signs included fever, abdominal pain and leukocytosis. Sinogram or percutaneous trans hepatic cholangiogram could help to confirm the diagnosis.
The consequence was more serious in HJ leak group, including more pancreatic fistula (36 vs. $11 \%, \mathrm{p}=0.019$ ), intra-abdominal abscess ( 36 vs. $11 \%, \mathrm{p}=0.017$ ), wound infection (21 vs. 4\%, $\mathrm{p}=0.023)$ and delayed gastric emptying ( 36 vs. $10 \%, \mathrm{p}=0.015$ ). Surgical interventions were required for nearly $2 / 3$ of patients. Abdominocentesis and postoperative percutaneous trans hepatic bile drainage (PTBD) were most frequently used. Abdominocentesis was easily to perform, but could not control the bile leak as effectively as combined with PTBD. PTBD was reported to be succeeded in healing of the postoperative HJ leak, even for the absence of intrahepatic biliary duct dilatation, but it was technique dependence sometimes. When the suspected infectious fluid was detected on CT scan and the existing drainage was insufficiency, the ultrasound guided percutaneous drainage was performed, and most leaks could heal. ${ }^{15}$

There are four reoperations in HJ leak group (29 vs. $6 \%$ in non-HJ leak group, $\mathrm{p}=0.010)$. ALL the 4 patients had "early" or "high-output" HJ leaks, and developed a deteriorated condition, even after aggressive percutaneous drainage. Two of them developed diffused peritonitis, and one patient died for septic shock even after the reoperation. The other two patients developed massive bleeding and received trans catheter arterial embolization (TAE) successfully. TAE was widely used as the first-line treatment choice for delayed massive bleeding after PD. And after hemostasis, both percutaneous catheter drainage and reoperation with drain replacement were feasible methods to eliminate the cause of bleeding. ${ }^{16}$ For HJ-leak associated massive bleedings, we preferred the latter, because the embolization of common hepatic artery would ruin the blood supply of HJ anastomoses which might result in delayed healing, anastomotic stenosis or liver abscess. Placement of Ttube drainage in reoperation could resolve these problems. If the patients recovered uneventful, the $\mathrm{T}$ tube will be removed within 2 weeks, but may be maintained for one month or more if the patients suffered for severe PF or abdominal infection.

In the 4 reoperations, the $\mathrm{HJ}$ anastomose defect were found at the anterior wall with a semicircle rupture. This would explain why the percutaneous drainages were unsuccessful.

\section{CONCLUSION}

Hepaticojejunostomy leaks are rare after PD. The complication severity ranges from trivial to life threatening. It may be associated with nutritional status, width of common bile duct and surgical procedures. Surgical interventions are required for more than half of HJ leaks. A good outcome can be expected.

Funding: No funding sources Conflict of interest: None declared

Ethical approval: The study was approved by the institutional ethics committee 


\section{REFERENCES}

1. Suzuki Y, Fujino Y, Tanioka Y, Ajiki T, Hiraoka K, Takada M, et al. Factors influencing hepaticojejunostomy leak following pancreaticoduodenal resection;importance of anastomotic leak test. Hepato-gastroenterology. 2003;50(49):254-7.

2. Burkhart RA, Relles D, Pineda DM, Gabale S, Sauter PK, Rosato EL, et al. Defining treatment and outcomes of hepaticojejunostomy failure following pancreaticoduodenectomy. J Gastrointest Surg. 2013;17(3):451-60.

3. Herzog T, Belyaev O, Bakowski P, Chromik AM, Janot M, Suelberg D, et al. The difficult hepaticojejunostomy after pancreatic head resection: reconstruction with a $\mathrm{T}$ tube. Am J Surg. 2013;206(4):578-85.

4. Antolovic D, Koch M, Galindo L, Wolff S, Music E, Kienle P, et al. Hepaticojejunostomy analysis of risk factors for postoperative bile leaks and surgical complications. J Gastrointest Surg. 2007;11(5):55561.

5. Herzog T, Belyaev O, Hessam S, Uhl W, Chromik AM. Management of isolated bile leaks after pancreatic resections. J Invest Surg. 2014;27(5):273-81

6. Herzog T, Belyaev O, Uhl W, Seelig MH, Chromik A. Hepaticojejunostomy after pancreatic head resection - technical aspects for reconstruction of small and fragile bile ducts with T-tube drainage. Zentralbl Chir. 2012;137(6):559-64.

7. Uppal S, Al-Niaimi A, Rice LW, Rose SL, Kushner DM, Spencer RJ, et al. Preoperative hypoalbuminemia is an independent predictor of poor perioperative outcomes in women undergoing open surgery for gynecologic malignancies. Gynecol Oncol. 2013;131(2):416-22.

8. Ionescu D, Tibrea C, Puia C. Pre-operative hypoalbuminemia in colorectal cancer patients undergoing elective surgery - a major risk factor for postoperative outcome. 2013;108(6):822-8.
9. Critselis E, Panagiotakos DB, Machairas A, Zampelas A, Critselis AN, Polychronopoulos E. Risk and predictive factors of hypoalbuminemia in cancer patients following extensive abdominal surgery despite total parenteral nutritional support. Int J Food Sci Nutr. 2012;63(2):208-15.

10. Iacono C1, Ruzzenente A, Campagnaro T, Bortolasi L, Valdegamberi A, Guglielmi A. Role of preoperative biliary drainage in jaundiced patients who are candidates for pancreatoduodenectomy or hepatic resection: highlights and drawbacks. Ann Surg. 2013;257(2):191-204.

11. Bhayani NH, Enomoto LM, James BC, Ortenzi G, Kaifi JT, Kimchi ET, et al. Multivisceral and extended resections during pancreatoduodenectomy increase morbidity and mortality. Surgery. 2014;155(3):567-74.

12. Ferrero A, Russolillo N, Viganò L, Sgotto E, Lo Tesoriere R, Amisano M, et al. Safety of conservative management of bile leakage after hepatectomy with biliary reconstruction. J Gastrointest Surg. 2008;12(12):2204-11.

13. Hoekstra LT, van Gulik TM, Gouma DJ, Busch OR., et al. Posthepatectomy bile leakage: how to manage. Dig Surg. 2012;29(1):48-53.

14. Hirono S, Kawai M, Tani M, Okada K, Miyazawa M, Shimizu A, et al. Indication for the use of an interposed graft during portal vein and/or superior mesenteric vein reconstruction in pancreatic resection based on perioperative outcomes. Langenbecks Arch Surg. 2014;399(4):461-71.

15. Righi D, Franchello A, Ricchiuti A, Breatta AD, Versace K, Calvo A, et al. Safety and efficacy of the percutaneous treatment of bile leaks in hepaticojejunostomy or split-liver transplantation without dilatation of the biliary tree. Liver Transpl. 2008;14(5):611-5.

16. Zhang J, Zhu X, Chen H, Qian HG, Leng JH, Qiu $\mathrm{H}$, et al. Management of delayed postpancreaticoduodenectomy arterial bleeding: interventional radiological treatment first. Pancreatology. 2011;11(5):455-63.

Cite this article as: Qiu $\mathrm{H}$, Zhang J, Qian HG, Leng JH, Wu JH, Liu BN, et al. Hepaticojejunostomy leak after pancreaticoduodenectomy. Int Surg J 2016;3:6038. 